\title{
Long term decline of P100 amplitude in migraine with aura
}

\author{
Nofal M Khalil, Nigel J Legg, Duncan J Anderson
}

\begin{abstract}
Objectives-To investigate visual function in migraine using visual evoked potentials.

Methods-Electroretinograms (ERGs) and visual evoked potentials (VEPs) to single flash (SF) and pattern reversal (PR) stimuli were studied in 92 migraine subjects and 62 controls.

Results-In subjects with migraine, ERGs to single flash were normal. Mean latencies of the P1 and P2 waves in the SFVEP were increased at the occiput by $6 \%$ and $4 \%$ respectively, but normal at the vertex. Mean latency of the P100 wave in the PRVEP was increased by $5 \%$. These increases were not related to the presence or absence of an aura or to the duration of migraine. P100 amplitude showed a more complex abnormality. It was increased in migraine without aura by $23 \%$ compared with controls, regardless of duration of migraine. In migraine with aura it was similarly increased, by $23 \%$, in cases of short duration, but in addition it showed a sharp decline with duration. In cases with a duration of 30 or more years it was $36 \%$ less than in cases of short duration, and $21 \%$ less than in controls.

Conclusions-Subjects with migraine have constitutionally prolonged VEP latencies and increased P100 amplitude, but the latter declines to below normal in cases with a long history of migraine with aura. This decline may reflect subtle neuronal damage within the visual system from repeated transient ischaemia experienced during the aura. Future electrophysiological and other studies will need to be controlled for duration of migraine history.
\end{abstract}

(F Neurol Neurosurg Psychiatry 2000;69:507-511)

Keywords: migraine; visual evoked potentials

Department of

Neurology, Imperial

College School of

Medicine,

Hammersmith

Hospital Campus, Du

Cane Road, London

W12 0NN, UK

N M Khalil

N J Legg

D J Anderson

Correspondence to: Dr Nigel J Legg

Received 22 November 1999 and in revised form

31 March 2000

Accepted 11 May 2000
The studies reported here formed part of a $\mathrm{PhD}$ project $^{4}$ in which visual function was studied in 92 subjects with migraine and 62 controls, by neurophysiological and psychophysical methods. Here we report the results of investigation by electroretinogram (ERG), SFVEP, and PRVEP. Some preliminary data have already been published. ${ }^{56}$

\section{Methods}

SUBJECTS

The patients in this study were not a random sample of the migraine population. They came from a wide age range, and there was a deliberate selection for those with a visual aura and with attacks precipitated by visual stimuli, to provide subgroups large enough for meaningful comparisons. Controls were found among colleagues, the spouses, or friends of subjects with migraine, and other volunteers. All subjects had a visual acuity of $6 / 6$ or better, and none of them had any visual disorder.

All 92 subjects fulfilled the International Headache Society (IHS) criteria for a diagnosis of migraine with or without aura. ${ }^{7}$ There were 72 women and 20 men, aged 16-59 years (mean 40.2 (SD 12.5) years). All were free of headache at the time of testing, and none were taking prophylactic treatment. Forty seven had migraine with aura (MA), 37 had migraine without aura (MO), and eight had both. Results from these eight patients have been included only when the whole migraine group is considered. All patients with MA had a visual aura, and seven also had other aura symptoms. Seventy three patients reported attacks triggered by visual stimuli, such as bright lights, flashing lights, or patterns. Migraine frequency varied between one or more attacks a week and 5-10 a year. Controls comprised 62 subjects, 44 women and 18 men, aged 17-58 years (mean 36.5 (SD 13.1)years). All participants were assessed using a headache questionnaire and clinical neurological examination, including fundoscopy, measurement of visual acuity, and assessment of visual fields and external ocular movements. The study was performed with approval from the ethics committee of the Hammersmith Hospital and the objectives of the tests were explained to all subjects at the beginning of the test session.

\section{TECHNIQUES}

Subjects were encouraged throughout the tests, to maintain their interest and concentration, and a break was taken for refreshment. The 11 control and 15 subjects with migraine who needed glasses wore them throughout all tests. All investigations were carried out with a 
Table 1 Flash ERG in controls and migraine subjects

\begin{tabular}{llll}
\hline & $\begin{array}{l}\text { Controls } \\
(n=62)\end{array}$ & $\begin{array}{l}\text { Migraine } \\
\text { subjects }(n=92)\end{array}$ & $\begin{array}{l}\text { p Value } \\
\text { (unpaired t test) }\end{array}$ \\
\hline Latency $(\mathrm{ms}):$ & & & \\
$\quad$ a Wave & $23.2(2.1)$ & $22.8(1.8)$ & 0.78 \\
$\quad$ b Wave & $49.1(5.9)$ & $48.9(5.3)$ & 0.83 \\
Amplitude $(\mu \mathrm{V}):$ & & \\
$\quad$ a Wave & $9.4(2.8)$ & $9.6(3.9)$ & 0.77 \\
$\quad$ b Wave & $29.9(12.0)$ & $27.4(9.3)$ & 0.22 \\
\hline
\end{tabular}

Values are mean (SD) of the averaged monocular responses.

background luminance of about $1 \mathrm{~cd} / \mathrm{m}^{2}$ as checked by a photometer.

Electroretinograms (ERGs) were recorded simultaneously to the single flash VEP (SFVEP) via infraorbital skin electrodes referred to the ipsilateral temple.

Single flash VEPs (SFVEPs) were elicited with binocular viewing using a PS100 (SLE) xenon flash tube (2 flashes/s; energy output 0.4 $\mathrm{J}$ at the source; flash duration $65 \mu \mathrm{s}$; distance from the eye $15 \mathrm{~cm}$ ). Averaging was started 5 seconds after the first flash to allow the pupils to adapt. Recordings were taken from electrodes placed at $\mathrm{Oz}$ and $\mathrm{Cz}$, using a linked ear reference. A total of 240 responses were averaged and displayed.

Pattern reversal VEPs (PRVEPs) were elicited using a chequerboard reversing at 2 /s displayed on a Medelec TV type visual stimulator at $100 \mathrm{~cm}$ distance. The stimulus field subtended $30 \times 25$ degrees of visual angle and the individual squares $0.63^{\circ}$. The mean luminance of the screen was $54.2 \mathrm{~cd} / \mathrm{m}^{2}$ and contrast was $95 \%$. Recordings were obtained from an electrode placed at $\mathrm{Oz}$, referred to a common reference at Fz. Binocular and monocular whole field tests were carried out; 240 responses were averaged and displayed for each test.

Statistical analysis was performed with Maxitab (IBM), Statwork, and Statview SE (Macintosh) statistical programmes. The differences between sets of data from subjects with migraine and control subjects were tested for significance by the unpaired $t$ test. The effects on the results of various factors in the migraine group were tested with analysis of variance (ANOVA) and simple and multiple regression analysis.

\section{Results}

We present all results for controls and migraineurs, and separately for migraine with and without aura when appropriate. No results showed any association with the presence or absence of visual triggers.

There were no significant differences between migraine subjects and controls nor between MA and $\mathrm{MO}$ in the latency or amplitude of the a or b waves in the flash ERG (table 1), and results did not vary with age, sex, or duration of migraine.

Single flash VEPs are very variable, and in some subjects the averaged responses were not clear enough for measurement. The number of subjects with readable responses is shown for each test (table 2). In subjects with migraine the P1 and P2 latencies at the occiput were increased by $6.4 \%$ and $3.8 \%$ respectively com-
Table 2 Single flash VEP responses in controls and migraine subjects at the occiput $(\mathrm{Oz})$ and vertex $(\mathrm{Cz})$ positions

\begin{tabular}{|c|c|c|c|c|}
\hline & \multicolumn{2}{|c|}{ Controls } & \multicolumn{2}{|c|}{ Migraine subjects } \\
\hline & $n t$ & mean $(S D)$ & $n t$ & mean $(S D)$ \\
\hline \multicolumn{5}{|c|}{ Latency (ms): } \\
\hline $\mathrm{Oz} \mathrm{P} 1$ & 51 & $71.6(10.7)$ & 75 & $76.2(10.2)^{\star}$ \\
\hline Oz P2 & 56 & $124.8(10.0)$ & 89 & $129.6(10.3)^{\star}$ \\
\hline Cz P1 & 41 & $74.5(11.9)$ & 64 & $77.1(12.9)$ \\
\hline $\mathrm{Cz}$ N2 & 49 & $120.4(11.1)$ & 81 & $121.4(10.2)$ \\
\hline \multicolumn{5}{|c|}{ Amplitude $(\mu \mathrm{V})$ : } \\
\hline $\mathrm{Oz} P 1$ & 51 & $6.9(5.9)$ & 75 & $7.6(4.6)$ \\
\hline Oz P2 & 56 & $13.8(6.3)$ & 89 & $14.0(6.9)$ \\
\hline Cz P1 & 41 & $5.6(3.5)$ & 64 & $5.6(3.3)$ \\
\hline $\mathrm{Cz} N 2$ & 49 & $11.7(5.0)$ & 81 & $10.3(4.7)$ \\
\hline
\end{tabular}

${ }^{\star} \mathrm{p}<0.001$; unpaired $t$ test.

†Number of subjects with readable responses.

Table 3 P100 latency in control and migraine subjects

\begin{tabular}{|c|c|c|c|c|}
\hline & \multicolumn{2}{|c|}{ Controls } & \multicolumn{2}{|c|}{ Migraine subjects } \\
\hline & $n$ & mean $(S D)$ & $n$ & mean $(S D)$ \\
\hline \multicolumn{5}{|l|}{ Latency $(\mathrm{ms})$ : } \\
\hline RE & 62 & $112.1(5.6)$ & 92 & $117.9(5.4)^{\star \star}$ \\
\hline $\mathrm{LE}$ & 62 & $113.1(5.5)$ & 92 & $118.8(5.3)^{\star \star}$ \\
\hline BIN & 62 & $110.5(5.8)$ & 92 & $115.2(5.6)^{\star \star}$ \\
\hline$M$ (av mon) & 18 & $113.8(5.9)$ & 20 & $120.0(6.2)^{\star}$ \\
\hline $\mathrm{F}$ (av mon) & 44 & $112.1(5.2)$ & 72 & $117.8(4.7)^{\star \star}$ \\
\hline
\end{tabular}

${ }^{\star} \mathrm{p}=0.007 ;{ }^{\star}{ }^{*} \mathrm{p}<0.001 ;$ unpaired $t$ test, two tailed. Values are mean (SD) PRVEP latencies recorded at Oz position. RE=Right eye; LE=left eye; BIN=binocular; $\mathrm{M}=$ male; $\mathrm{F}=$ female; av mon=averages of RE and LE monocular.

pared with controls. There were no increases at the vertex. The P1 and P2 amplitudes were similar in migraine subjects and controls. There were no differences between MA and $\mathrm{MO}$, and the results did not vary with age, sex, or duration of migraine.

In the PRVEP the P100 latency was 5\% longer in male and female subjects with migraine than in controls, with binocular and monocular whole field stimulation, at all recorded positions. This increase in latency was comparable with those of the occipital P1 and P2 latencies in the SFVEP and was independent of age or duration of migraine. Latencies were shorter in women than men, and the difference was just significant in subjects with migraine (controls $t=1.12$, $\mathrm{p}=0.269$; migraine $t=2.13, \mathrm{p}=0.036)$. Average monocular and binocular P100 latency results recorded at $\mathrm{Oz}$ are presented in table 3.

Results for P100 amplitude are shown in table 4. Amplitude was slightly increased in the migraine group as a whole. When subjects were separated into MA and MO groups, amplitude in MO was $23 \%$ higher than in controls but in MA it was similar to controls. Amplitudes in MA were inversely correlated with duration of migraine but there was no such correlation in MO. Amplitudes in MA of duration $\leqslant 10$ years were the same as those in the MO group and $23 \%$ higher than controls. Those in MA of duration $\geqslant 30$ years were $36 \%$ lower than in the short duration group and also $21 \%$ lower than controls. Patients with a long duration of migraine were of course older than those with short duration, but multiple regression analysis showed that the reduction in P100 amplitude in MA was correlated with duration and not with age ( $r=0.60$; duration: $t=3.35, \mathrm{p}=0.002$; 
Table $4 \quad$ P100 amplitude in controls and migraine subjects

\begin{tabular}{|c|c|c|c|c|c|c|c|c|}
\hline & \multirow[b]{3}{*}{$n$} & \multirow{3}{*}{$\begin{array}{l}\text { Amplitude }(\mu V) \\
(\text { mean }(S D))\end{array}$} & \multicolumn{6}{|c|}{ Significance of difference from: } \\
\hline & & & \multicolumn{2}{|l|}{$C$} & \multicolumn{2}{|l|}{$M O$} & \multicolumn{2}{|c|}{$M A \leqslant 10 y$} \\
\hline & & & $t$ & $p$ Value & $\bar{t}$ & $p$ Value & 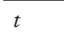 & $p$ Value \\
\hline $\mathrm{C}$ & 62 & $15.1(4.7)$ & & & & & & \\
\hline M & 92 & $16.8(5.1)$ & 1.99 & 0.048 & & & & \\
\hline MO & 37 & $18.6(5.3)$ & 3.40 & 0.001 & & & & \\
\hline MA & 47 & $15.2(4.4)$ & 0.05 & 0.96 & 3.25 & 0.0017 & & \\
\hline$M A<10 y$ & 17 & $18.6(3.1)$ & 2.91 & 0.0048 & 0.03 & 0.98 & & \\
\hline $\mathrm{MA}>30 \mathrm{y}$ & 14 & $12.0(3.0)$ & 2.40 & 0.018 & 4.42 & 0.0001 & 6.06 & $<0.0001$ \\
\hline
\end{tabular}

$\mathrm{C}=$ Control subjects; $\mathrm{M}=$ all migraine; $\mathrm{MO}=$ migraine without aura; $\mathrm{MA}=$ migraine with aura $\leqslant 10 \mathrm{y}$ $=$ duration 10 years or less; $\geqslant 30 \mathrm{y}=$ duration 30 years or more. Amplitudes are the average of the monocular responses recorded at $\mathrm{Oz}$.

age: $t=0.02, \mathrm{p}=0.99)$. Amplitudes were not correlated with age in controls nor in MO.

\section{Discussion}

The most striking findings reported here were in P100 amplitude. Other studies of this ${ }^{3-13}$ have produced differing results, and this is understandable in the light of the present finding, of two trends acting in opposite directions. Simple comparison of our subjects with migraine and control subjects disclosed only a small difference. However, in MO, regardless of duration, and in MA of short duration the mean P100 amplitude was increased compared with controls by more than $20 \%$. In migraine with aura the drop in P100 amplitude with duration, comparing durations of 10 years or less with those of 30 years or more, was more than $33 \%$. These findings, which have not previously been reported, need two separate explanations. We think that the high P100 amplitude represents a constitutional abnormality of subjects with migraine, and that the low amplitude in MA of prolonged duration is an acquired phenomenon, related to the events of the aura.

The high constitutional amplitudes accord well with the proposition of Avicenna (see Isler $^{14}$ ) that migraine is due to "hyperexcitability" of the brain, which is extra sensitive to light and other stimuli, and also with the frequent complaint by subjects with migraine of a general intolerance of light, patterns, and flashing lights, even when they are not having an attack.

Since our study was performed Schoenen et $a l^{11}$ have shown that high P100 amplitudes recorded in subjects with migraine by conventional averaging of 250 stimuli, as in our method, were the result of potentiation. Amplitudes averaged from the first 50 stimuli were similar in subjects with migraine and controls, but after 250 stimuli normal subjects showed habituation, with a fall in amplitude, and subjects with migraine showed potentiation, with a rise. Amplitudes from the first 50 stimuli were similar in controls and those with migraine but in a subsequent study they tended to be lower in subjects with migraine $(p=0.068) .{ }^{15}$ Studies of auditory evoked potentials have shown an increase in the amplitude of responses with increased stimulus intensity which is steeper in subjects with migraine than controls. ${ }^{16}$ Welch et $a l^{17}$ have produced direct evidence of central neuronal hyperexcitability in migraineurs with aura by magnetoencephalography and NMR spectroscopy. They found large amplitude waves both during and between migraine attacks which they considered to be spontaneous neuronal discharges, and a high turnover of high energy phosphates. Several attempts have been made to resolve the question of cortical hyperexcitability by the use of transcranial magnetic stimulation. Over the visual cortex this can produce illusions of light (phosphenes) and over the motor cortex it can elicit motor evoked potentials (MEPs). Over the visual cortex results in MO have been the same as controls. ${ }^{18}{ }^{19}$ In unselected patients with MA phosphenes were fewer than in controls, and thresholds were the same. ${ }^{18}$ In MA with visual triggers phosphenes were increased and thresholds were lower than in controls. ${ }^{19}{ }^{20}$ The MEPs expressed as a fraction of compound muscle action potentials elicited by peripheral nerve stimulation were higher in both MO and MA than in controls. ${ }^{21}$ Motor thresholds in MO and MA both at rest and during isometric muscle contraction were the same as in controls; however, in cases of MA with a sensorimotor aura these thresholds were increased during isometric muscle contraction. ${ }^{22}$ In one study the proportionate increase in MEPs in migraine was correlated with attack frequency. ${ }^{21}$ Thus, at present no firm conclusions can be drawn from studies with these methods. Perhaps cortical excitability, hyperexcitability, habituation, and potentiation are not consistent over the whole of the cortex. An oral presentation during the proof stage of our paper gives some support to this suggestion (Stewart L, Walsh V, Rothwell J. Non-correlation of phosphene and motor thresholds: implication for TMS studies. Presented at meeting of the British Society for Clinical Neurophysiology, Cambridge, 9 June 2000).

Chronicle and Mulleners ${ }^{23}$ suggested that such hyperexcitability might be due to loss of inhibitory interneurons in the visual cortex, acquired as a result of the migraine attacks or their medication. This suggestion is incompatible with our data. If the phenomenon were acquired it should become more manifest with a longer duration of migraine, whereas it does not change in MO and it becomes less in MA. Furthermore it ought to be more obvious in patients with frequent attacks. We examined P100 amplitude for a relation with attack frequency but did not find one.

For the same reasons we regard the increased latencies of the P100 and of the SFVEP as constitutional. These increases, which are of the order of $5 \%$, are by no means as striking as the P100 amplitude findings, and perhaps less interesting. Most previous studies of any size have confirmed prolongation of P100 latency $^{324}$ but normal latencies have been reported in smaller surveys ${ }^{911}{ }^{13}$ Previous reports about SFVEP results have been conflicting or ambiguous. In the current study the occipital P1 and P2 latencies were both prolonged, but when only 51 migraineurs and 30 controls had been studied ${ }^{6}$ the prolongation of P1 was not significant. 
The basis for the prolonged latencies is unclear. Kennard et $a l^{3}$ suggested that they might have a structural basis, due to ischaemic damage during repeated attacks. If this were so a relation would be expected between latency and duration of migraine, which we did not find. Also, if relative cerebral ischaemia during a migraine aura was considered to be the cause the abnormalities should be confined to MA, whereas our findings were the same in MA and MO. The possibility of ergotamine effects could still be considered, but if these were being exerted by recurrent ischaemia it would be expected that the change would be related to duration of migraine.

There is a single case report ${ }^{26}$ of an increase in P100 latency, associated with reduced visual acuity, in a man taking daily ergotamine continuously for several years, with recovery to normal when the drug was stopped. We do not think this has any bearing on our patients. Many of them had taken occasional ergotamine for individual attacks, but none were taking it consistently. We therefore conclude that the prolonged latencies are constitutional, perhaps due to synaptic delay.

Abnormalities in 5-hydroxytryptamine have long been associated with migraine, and in phenylketonuria, which causes reduced brain concentrations of catecholamines and 5-hydroxytryptamine, PRVEP latency is prolonged, but can be normalised by dietary phenylalanine restriction. ${ }^{27}$ However in this condition P100 amplitudes are reduced. ${ }^{28}$

The fall in P100 amplitude with increasing duration of migraine in MA has not previously been seen, and has rarely been looked for. Correlations with migraine duration have been looked for in SFVEPs, RFVEPs, and PRVEPs, ${ }^{24}{ }^{29}{ }^{30}$ but the range of duration in all these studies was fairly small, and in some ${ }^{29} 30$ the number of subjects with MA was also small. Doubtless the relation has only been recognised in the present study because of the large number of subjects with MA and their wide range of duration of migraine.

The fall in amplitude could represent a loss of potentiation. We have looked for this in a few cases of MA with durations of $\leqslant 10$ years $(n=5)$ and $\geqslant 20$ years $(n=8)$ but in neither group were we able to reproduce the potentiation found by Schoenen et al. ${ }^{11}$

The cause of the reduced P100 amplitude in chronic MA is not immediately apparent. Stroke is known to occur with greater than normal frequency in subjects with migraine, especially those with MA. Routine head scanning did not form part of this study but no patient had had a clinical stroke, nor did they have either visual or other evidence for any major cerebral infarct. Nevertheless, ischaemia at a minor, more diffuse level, must be a likely aetiological candidate. The most obvious fact is that the cause is to be sought in the events of the aura itself, not in the mechanisms of headache or associated symptoms, as amplitude is not reduced in longstanding MO. Clinically, the visual aura consists of both positive symptoms, in the form of bright spots or twinkling lights, and negative ones in the form of scotomas, small multiple ones or a single, dense, enlarging one. These may well represent the underlying events of spreading depression, with a band of transient depolarisation followed by a more prolonged period of hyperpolarisation, and hence cortical inactivity, although the aura itself rarely consists of such a neat sequence, and at any moment often includes both positive and negative symptoms throughout the visual field. Nevertheless, the hypothesis of spreading depression accords well with the findings of changes in cerebral blood flow during an attack.

Originally cerebral blood flow was reported to fall by about $20 \%$ during attacks of MA, ${ }^{31} 32$ reaching $40-50 \mathrm{ml} / 100 \mathrm{~g} / \mathrm{min}$. This could well be appropriate to the reduced level of metabolic requirements in cortical spreading depression, but is well above the neural threshold for ischaemia. However, recalculation of the data allowing for the influence of scattered radiation has suggested that flow might have dropped to $16-23 \mathrm{ml} / 100 \mathrm{~g} / \mathrm{min}$ in the least perfused areas. ${ }^{33}$ This degree of ischaemia could certainly account for transient neurological deficits and possibly persistent deficits if the damage were repetitive. The neural damage caused by profound cerebral hypoxia is well known, but experimentally less severe transient hypoxia can also lead to permanent damage, if repeated over a long period..$^{34}$ It could therefore explain the fall in P100 amplitude we describe. However there is still conflict about the correct interpretation of cerebral blood flow results, and the extent of the fall during attacks of MA.

Another possible cause of ischaemia would be recurrent ergotamine intake, but this should produce the same results in MA and MO unless a combined effect of flow reduction associated with the aura and the vasoconstrictive action of the drug is postulated. In the case reported by Heider et $a l^{26}$ chronic ergotamine misuse led to an increase in P100 latency but did not reduce P100 amplitude.

If the primary cause of the amplitude change is ischaemia then the final pathway for cell damage might be a neurotoxic effect from the action of excitatory aminoacids, which can cause neuronal damage through prolonged activation of N-methyl-D-aspartate receptors. ${ }^{35}$ This process has been implicated in the pathophysiology of cell loss in ischaemia, but also in other situations, and might be relevant to impaired neuronal function in chronic MA.

Our results have sufficiently demonstrated a constitutional 20\% increase in P100 amplitude in MO and in MA of short duration. They also provide the first demonstration of an electrophysiological measure which declines progressively with duration of disease in MA, a change we tentatively attribute to repetitive transient ischaemia occurring during the aura. Despite numerous publications there has been no previous study which has in our view satisfactorily shown adverse effects on brain structure or function as a result of repeated migraine attacks. We suggest that duration of disease must be taken into account when assessing visual and other functions in migraine. 
1 Golla F, Winter A. Analysis of cerebral responses to flicker in patients complaining of episodic headache. Electroencephapatients complaining of episodic headach
logr Clin Neurophysiol 1959;11:539-49.

2 Richey EK, Kooi KA, Waggoner RW. Visually evoked responses in migraine. Electroencephalogr Clin Neurophysiol 1966;21:23-7.

3 Kennard C, Gawel M, Rudolph N, et al. Visual evoked potentials in migraine subjects. Research and clinical studies in headache 1978;6:73-80.

4 Khalil N. Investigations of visual function in migraine using visual evoked potentials and psychophysical tests [PhD thesis]. London: University of London, 1991.

5 Khalil N, Oozeer R, Legg N. Visual evoked potentials and contrast sensitivity function in migraine. Electroencephalogr Clin Neurophysiol 1988;70:132.

6 Khalil N, Legg N. Pathophysiology of migraine: a study using VEP and contrast sensitivity. In: Clifford Rose F, ed. New advances in headache research: Proceedings of the 7 th Migraine Trust International Symposium. London: SmithMigraine Trust Internati

7 Headache Classification Committee of the International Headache Society. Classification and diagnostic criteria for headache disorders, cranial neuralgias and facial pain. Cephalalgia 1988;8(suppl 7):1-96.

8 Rossi LN, Pastorino GC, Bellettini G, et al. Pattern reversal visual evoked potentials in children with migraine or tension-type headache [see comments]. Cephalalgia 1996; 16:104-6.

9 Benna P, Bianco C, Costa P, et al. Visual evoked potentials and brainstem auditory evoked potentials in migraine and transient ischemic attacks. Cephalalgia 1985;5(suppl 2): 53-8.

10 Drake M, Pakalnis A, Hietter S, et al. Visual and auditory evoked potentials in migraine. Electromyogr Clin Neurophysiol 1990;30:77-81.

11 Schoenen J, Wang W, Albert A, et al. Potentiation instead of habituation characterises visual evoked potentials in migraine patients between attacks. Eur f Neurol 1995;2: migraine

12 Sener HO, Haktanir I, Demirci S. Pattern-reversal visual evoked potentials in migraineurs with or without visual evoked potentials in migraineur
aura. Headache 1997;37:449-51.

13 Shibata K, Osawa M, Iwata M. Simultaneous recording of pattern reversal electroretinograms and visual evoked potentials in migraine [see comments]. Cephalalgia 1997; 17:742-7.

14 Isler H. Retrospect: the history of thought about migraine from Areteus to 1920. In: Blau J, ed. Migraine: clinical, therapeutic, conceptual and research aspects. London: Chapman and Hall Medical, 1987:659-74.

15 Afra J, Cecchini AP, De Pasqua V, et al. Visual evoked potentials during long periods of pattern-reversal stimulation in migraine Brain 1998;121:233-41.

16 Wang W, Timsit Berthier M, Schoenen J. Intensity dependence of auditory evoked potentials is pronounced in migraine: an indication of cortical potentiation and low serotonergic neurotransmission? Neurology 1996;46:14049.

17 Welch KM, Barkley GL, Ramadan NM, et al. NMR spectroscopic and magnetoencephalographic studies in migraine with aura: support for the spreading depression hypothesis. Pathol Biol Paris 1992;40:349-54

18 Afra J, Mascia A, Gerard P, et al. Interictal cortical excitability in migraine: a study using transcranial magnetic stimulation of motor and visual cortices. Ann Neurol 1998;44: 209-15.

19 Aurora SK, Ahmad BK, Welch KM, et al. Transcranial magnetic stimulation confirms hyperexcitability of occipital cortex in migraine. Neurology 1998;50:1111-4

20 Aurora SK, Cao Y, Bowyer SM, et al. The occipital cortex is hyperexcitable in migraine: experimental evidence. Headache 1999;39:469-76.

21 van der Kamp W, Maassen VanDenBrink A, Ferrari MD, et al. Interictal cortical hyperexcitability in migraine patients demonstrated with transcranial magnetic stimulation. $\mathcal{f}$ Neurol Sci 1996;139:106-10.

22 Maertens de Noordhout A, Pepin JL, Schoenen J, et al. Percutaneous magnetic stimulation of the motor cortex in migraine. Electroencephalogr Clin Neurophysiol 1992;85:11015.

23 Chronicle E, Mulleners W. Might migraine damage the brain? Cephalalgia 1994;14:415-18.

24 Winter A. Electrophysiological studies of the visual system of people with classical migraine, [PhD thesis]. University of Surrey, 1985.

25 Polich J, Ehlers CL, Dalessio DJ. Pattern-shift visual evoked responses and EEG in migraine. Headache 1986;26:451-6.

26 Heider W, Berninger T, Brunk G. Electro-ophthalmologic and clinical studies in chronic ergotamine abuse. Fortschrift der Ophthalmologie 1986;83:539-41.

27 Schafer EW, McKean CM. Evidence that monoamines influence human evoked potentials. Brain Res 1975;99:4958.

28 Jones SJ, Turano G, Kriss A, et al. Visual evoked potentials in phenylketonuria: association with brain MRI, dietary state, and IQ. F Neurol Neurosurg Psychiatry 1995;59:260-5.

29 Lehtonen JB. Visual evoked cortical potentials for single flashes and flickering light in migraine. Headache 1974;14: $1-12$.

30 Jonkman EJ, Lelieveld MH. EEG computer analysis in patients with migraine. Electroencephalogr Clin Neurophysiol 1981;52:652-5.

31 Olesen J, Larsen B, Lauritzen M. Focal hyperemia followed by spreading oligemia and impaired activation of $\mathrm{rCBF}$ in classic migraine. Ann Neurol 1981;9:344-52.

32 Lauritzen M, Skyhoj Olsen T, Lassen NA, et al. Changes in regional cerebral blood flow during the course of classic migraine attacks. Ann Neurol 1983;13:633-41.

33 Skyhoj Olsen T, Friberg L, Lassen NA. Ischemia may be the primary cause of the neurologic deficits in classic migraine. Arch Neurol 1987;44:156-61.

34 Brierly M, Graham D. Hypoxia and vascular disorders of the central nervous system. In: Adams J, Corsellis J, Duchen L, eds. Greenfields neuropathology. London: Arnold, 1984:125-

35 Rothman SM, Olney JW. Excitotoxicity and the NMDA receptor. Trends Neurosci 1987;10:299-302. 